\title{
ERRATA
}

\section{Erratum to: Synthesis and Biological Activity of Triacetonamine}

\author{
M. N. M. Yousif ${ }^{a, *}$, H. A. Soliman ${ }^{a}$, M. M. Said ${ }^{b}$, N. A. Hassan ${ }^{a, c}$, and F. M. E. Abdel-Megeid ${ }^{a}$ \\ ${ }^{a}$ Photochemistry Department, Chemical Industries Research Division, National Research Center, \\ Dokki, Cairo, 12622 Egypt \\ ${ }^{b}$ Pharmaceutical Chemistry Department, Faculty of Pharmacy and Drug Manufacturing, \\ Pharous University in Alexandria, Alexandria, 12622 Egypt \\ ${ }^{c}$ Pharmaceutical Science Department, College of Pharmacy, Shaqra University, Riyadh, 11671 Saudi Arabia \\ *e-mail:mahmoud_nabil18@yahoo.com
}

Received May 6, 2019; revised May 12, 2020; accepted May 12, 2020

DOI: $10.1134 / \mathrm{S} 1070363220040350$

Supplementary materials are available for this article from the authors.

The original article can be found online at https://doi.org/10.1134/S1070363220030202 